\title{
Programa barrios de Quito (UCE): entre la pandemia y el desarrollo de la virtualidad
}

PhD. Santiago Morales Cardoso

smorales@uce.edu.ec

MSc. Diana Villamar Flores devillamarf@uce.edu.ec

MSc. Jorge Antonio Piedra Rosales

japiedra@uce.edu.ec

https://orcid.org/0000-0002-0379-1173

MSc. Luis Muisin

luis.muisin4336@utc.edu.ec

\section{RESUMEN}

La educación en línea o capacitación a través de Internet conocido en el idioma inglés como e-learning comprende una combinación de recursos, interactividad, apoyo y actividades de aprendizaje estructuradas. Para realizar todo este proceso es necesario conocer las innumerables posibilidades y también algunas limitaciones que el soporte informático en plataformas virtuales nos ofrece. En la más reciente vivencia de pandemia que atravesó el mundo y esperando que sea la última, surge la creatividad del ser humano y se logra integrar esta iniciativa con la responsabilidad universitaria de Vinculación con la Sociedad, fortaleciendo un importante trabajo ya iniciado en el Programa "Barrios de Quito" de la Universidad Central del Ecuador, surge la propuesta en pandemia y logramos sostener un soporte permanente a la ciudadanía, contribuyendo con otros tipos de conocimientos que complementan el aporte social que ha venido generando este programa. Conseguimos que docentes (40) y estudiantes (500) de varias facultades y carreras compartan sus conocimientos multidisciplinarios reforzando las competencias profesionales de los estudiantes y permitiendo al mismo tiempo resolver problemas complejos de los individuos en el ámbito laboral, social y familiar. Mediante este trabajo se logró la implementación de una herramienta virtual de capacitaciones dentro del ecosistema informático de la universidad en donde los involucrados se empoderaron de esta posibilidad tecnológica permitiendo un gran progreso en los procesos de vinculación, 
generando nuevos temas de interés social, de conocimiento humano, salud y de conciencia con el medio ambiente, implementación de procesos de investigación, capacitación, los cuales se han adecuado a los parámetros de la virtualidad y han apoyado en momentos críticos al avance individual y colectivo de nuestros vecinos de los barrios del Distrito Metropolitano de Quito.

Palabras Clave: plataformas virtuales; vinculación con la sociedad; ecosistema informático; capacitación; virtualidad 


\title{
Barrios de Quito program (UCE): between the pandemic and the development of virtuality
}

\begin{abstract}
Online education or training through the Internet known in the English language as elearning comprises a combination of resources, interactivity, support and structured learning activities. To carry out this entire process, it is necessary to know the innumerable possibilities and also some limitations that computer support on virtual platforms offers us. In the most recent experience of a pandemic that crossed the world and hoping that it will be the last, the creativity of the human being arises and it is possible to integrate this initiative with the university responsibility of Linking with Society, strengthening an important work already started in the Program " Barrios de Quito "of the Central University of Ecuador, the proposal arises in a pandemic and we managed to sustain permanent support to citizens, contributing with other types of knowledge that complement the social contribution that this program has been generating. We get teachers (40) and students (500) from various faculties and careers to share their multidisciplinary knowledge, reinforcing the professional competencies of students and at the same time allowing complex problems of individuals to be solved in the workplace, social and family. Through this work, the implementation of a virtual training tool within the university's computer ecosystem was achieved, where those involved were empowered by this technological possibility, allowing great progress in the linking processes, generating new topics of social interest, of human knowledge., health and awareness with the environment, implementation of research processes, training, which have been adapted to the parameters of virtuality and have supported at critical moments the individual and collective progress of our neighbors in the neighborhoods of the Metropolitan District of Quito.
\end{abstract}

Key Words: virtual platforms; link with society; information technology ecosystem; training; virtuality

Artículo recibido: 15 enero 2022 Aceptado para publicación: 08 febrero 2022

Correspondencia: smorales@uce.edu.ec Conflictos de Interés: Ninguna que declarar 


\section{INTRODUCCIÓN}

La pandemia del último año generada por el virus del SARS-CoV-2 conocida como el COVID 19, ha pausado los eventos presenciales en el país y en el mundo en general. Las actividades de Vinculación con la Sociedad desde la universidad ecuatoriana no fueron la excepción y debieron ser reinventadas, así es que el Programa Barrios de Quito llevado a cabo desde la Universidad Central del Ecuador desde el año 2018 cuenta con varios proyectos en diferentes áreas relacionadas con lo psicológico, salud, social entre otros, los cuales se han adecuado bajo los parámetros de la virtualidad con un gran éxito.

Es fundamental que la educación esté al servicio de políticas sociales públicas destinadas a construir y consolidar un modelo de desarrollo social, motivo por el cual se vinculó el e-learning, integrando la educación en línea y el uso de las Tecnologías de Información (TIC) además de otros elementos didácticos para el aprendizaje y la enseñanza, lo que complementa la estrecha relación entre el impulso de la divulgación de la información y la necesidad de la sociedad por aprender.

Las temáticas que se abarcan se presentan en la Tabla 1, y son de diferentes índoles las mismas que son trabajadas desde las diferentes carreras de la universidad.

Tabla 1 - Proyectos en Plataforma. Programa Barrios de Quito

\begin{tabular}{|c|c|}
\hline CARRERA & TEMA PROYECTO \\
\hline Radiología & $\begin{array}{l}\text { - Cáncer de mama, Importancia de la mamografía en el } \\
\text { diagnóstico temprano de cáncer de mama }\end{array}$ \\
\hline Enfermería & - Transfórmate Mujer \\
\hline Trabajo Social & $\begin{array}{l}\text { Participación y liderazgo barrial (Liderazgo - Educación } \\
\text { Popular - Lideresas barriales- Enlazando Redes). } \\
\text { - Reduce, Reúsa y Recicla desechos orgánicos e inorgánicos } \\
\text { "Por mi hogar" } \\
\text { - Vivamos sin corrupción } \\
\text { - Creación y funcionamiento de la Plataforma Virtual } \\
\text { "Barrios de Quito" } \\
\text { - Levantamiento de datos socio económicos del D. M. Quito } \\
\text { 2017-2020 } \\
\text { - Relaciones intrafamiliares en Pandemia Covid-19 }\end{array}$ \\
\hline $\begin{array}{l}\text { Facultad de Comunicación } \\
\text { Social e Ing. Química }\end{array}$ & - Yo Emprendo \\
\hline Jurisprudencia & - Justicia y Equidad \\
\hline $\begin{array}{l}\text { Filosofía, Letras y Ciencias } \\
\text { de la Educación. }\end{array}$ & - Educación Inclusiva \\
\hline $\begin{array}{l}\text { Vicerrectorado de } \\
\text { Investigación, Innovación y } \\
\text { Doctorados }\end{array}$ & - Huella Juvenil - Innovación Juvenil 2030 \\
\hline Química & - Manejo adecuado de alimentos \\
\hline
\end{tabular}


Nos planteamos como Objetivo el "Configurar un entorno de difusión tecnológica para que a través de este se lleven a cabo la generación de contenido y las respectivas capacitaciones virtuales que se pueden dar en el programa Barrios de Quito, dentro de las actividades de vinculación con la sociedad establecidas en cada una de las facultades que conforman el programa".

\section{Así mismo nos propusimos acentuar el trabajo sobre la base de los siguientes objetivos} específicos:

- Evaluar diferentes plataformas e-learning que permitan mantener una educación en línea con los estándares que cada capacitación lo necesite.

- Configurar la plataforma seleccionada con el fin de facilitar el uso a los capacitadores de los diferentes proyectos en el programa Barrios de Quito.

- Capacitar a las y los diferentes estudiantes de cada carrera que hagan uso de la plataforma, para poder mantener cada proyecto organizado antes, durante y después de su tiempo de capacitación y llegar de forma eficaz hacia cada uno de los diferentes usuarios del programa Barrios de Quito.

- Dar soporte continuo y permanente en cada uno de los proyectos para su buen manejo o para alguna actividad adicional que requiera el grupo de programas de Barrios de Quito.

En medio de la pandemia surgieron diferentes ideas, las mismas que fueron discutidas y resueltas sobre la base de teorías que permitan mejorar las condiciones de vida de la población, motivo por el cual recurridos al Libro Verde de la Innovación de la Comisión Europea (1995) donde se expone que la innovación se considera como sinónimo de producir, asimilar y explotar con éxito una novedad, en las esferas económica y social, de forma que aporte soluciones inéditas a los problemas y logre responder a las necesidades de las personas y de la sociedad.

El proceso de innovación estaría compuesto por dos vertientes: la primera, especializada en el conocimiento, mientras que la segunda se dedica fundamentalmente a su aplicación, para llegar a convertir las ideas que surgen de ese conocimiento en un proceso, un producto o un servicio que incorpore nuevas ventajas para el mercado. (HERNÁNDEZASCANIO, 2016). Dentro de este proceso de innovación las plataformas de aprendizaje virtual tienen un rol importante, ya que resultan ser un programa informático útil para administrar un curso en línea, además el término de aula virtual es precisamente una muy 
buena analogía que se le puede conferir a la plataforma de aprendizaje virtual con el aula presencial o tradicional pues es donde ocurren los procesos académicos y de enseñanza sin la necesidad de compartir un mismo espacio físico y temporal. Mora-Vicarioli, F. (2019).

En los últimos años la educación en línea se ha expandido y consolidado ampliamente en todos los niveles educativos y en situaciones formales y no formales de aprendizaje, (Salvat, 2018), la eficacia del e-learning según (Kizilcec, 2017) radica en el establecimiento de metas y la planificación estratégica para lograr los objetivos del curso sin embargo (Van Laer, 2017) muestran como los alumnos en los entornos de aprendizaje en línea tienden a fracasar por su falta de autonomía. Por lo que (Yen, 2016), llama la atención sobre la necesidad de incorporar procesos para facilitar la autorregulación del aprendizaje, dado que los aprendices pueden tener dificultades para formular sus objetivos, prever y planificar sus actividades. El aprendizaje autorregulado hace referencia a la toma de decisiones que permiten al estudiante controlar su proceso de aprendizaje, orientado al logro de las metas en un contexto que posee condiciones específicas. (Moneta Pizarro, 2018). Por la parte del docente o encargado del curso, se requiere una visión más flexible del aprendizaje y los medios para alcanzar eficazmente ese proceso en el alumnado (Verdezoto Rodríguez, 2018); en este caso la plataforma elearning según (Galvis, 2016) “posibilita la vinculación a una comunidad virtual mediante el intercambio de preguntas, dudas, respuestas, documentos, entre alumnado y profesorado, haciéndoles partícipes de la vida que puede generar dicha comunidad." La relación que tradicionalmente se imponía de forma vertical y unidireccional entre docentes y estudiantes evoluciona hacia un modelo más horizontal. También el sistema de evaluación tradicional deberá adecuarse a las nuevas tendencias sin perder su importancia para medir el aprendizaje de los educandos. (Gallegos Elias, 2021)

Se define el aprendizaje virtual de un estudiante para alcanzar los conocimientos relacionados con el grado de Economía mediante el mapa que relaciona la consecución de competencias mediante contenidos y viceversa. (Requena, 2004)

La evaluación e-learning se ha abordado desde diferentes flancos, desarrollándose enfoques de evaluación de aprendizaje virtual en términos socioeconómicos, tecnológicos, educativos (pedagógicos), metodológicos y psicológicos. (Bravo, 2006) 
Aprovechando las TIC's han surgido nuevas formas de producir, almacenar, distribuir y retroalimentar información generando cambios muy importantes en el proceso educativo promoviendo así mayores niveles de acumulación de capital humano y por ende de un mejor crecimiento y desarrollo sociocultural. (Moreno, 2016)

Valiéndonos del e-learning pretendemos abarcar dos temas puntuales, el primero hace referencia a la prevención del cáncer de mama, Tovato J (2013) impartió un curso virtual para enfermeras y descubrió que se necesitaban métodos innovadores para lograr un enfoque educativo eficaz para enseñar contenido relacionado con el cáncer de mama, análogamente Magaña Valladares (2018) utilizó la plataforma Moodle como sistema de gestión del aprendizaje para capacitación de profesionales de la salud en la prevención de cáncer de mama. En el caso de manejo de desechos tenemos que la implementación de una plataforma Moodle evidencia un proceso de enseñanza mediado por herramientas tecnológicas que impulsa en los estudiantes el manejo adecuado de residuos peligrosos al cabo de desarrollo de prácticas experimentales. (Ramos, 2021)

En la actualidad se aplican diferentes metodologías virtuales, utilizando herramientas y plataformas de aprendizaje virtual que han proporcionado la inserción de la tecnología en el que hacer educativo para el aprendizaje, entre estas metodologías emergentes tenemos por ejemplo, en Ecuador, Basantes, Naranjo y Ojeda (2018) identificaron el potencial de la metodología PACIE (Presencia, Alcance, Capacitación, Interacción, E-learning) vista desde un enfoque mixto fortalece el aprendizaje, vigorizando los cánones curriculares online, promoviendo los valores humanos junto a los estudiantes y estimulando el trabajo en equipo. Y si hablamos de herramientas y medios virtuales tenemos casos como, por ejemplo, en Colombia donde De-La-Hoz y Fontalvo (2019) utilizando la plataforma Moodle, desarrollaron una metodología para clasificar y predecir la frecuencia y tiempo de permanencia de los usuarios en ambientes virtuales de educación, y el desempeño en sus evaluaciones, implementando un análisis de clúster que permitió la identificación de grupos. Fernández-Bedoya, V. H. (2021).

Existen diferentes entornos virtuales de aprendizaje, y uno de los más ampliamente utilizados es Moodle el cual se distribuye como un software libre. Es una plataforma para la creación de cursos y sitios web basados en Internet. Como sistema de gestión de contenidos se centra en la creación y actualización de cursos para profesores y 
estudiantes. Constituye un recurso para facilitar el aprendizaje, trabajo en grupos y colaboración en proyectos. (Pérez Pérez, 2020)

\section{METODOLOGÍA}

Dentro de cualquier proyecto de tecnología es importante que se lo lleve a cabo en forma metodológica y en esta oportunidad se aplicaron las siguientes fases:

FASE 1: Se realizó una evaluación de las diferentes plataformas existentes en el mercado que podían ofrecer cursos de capacitación, considerando parámetros que consideramos relevantes:

- Configuración rápida.

- Fácil acceso.

- Cantidad de usuarios que pueden interactuar en la plataforma.

- Comunicación e interacción entre los participantes (chats, foros, correos)

- Introducción de módulos y cursos con facilidad y factibilidad.

FASE 2: Debido a las limitaciones económica se define que la plataforma sea de código abierto para que se la pueda configurar con las especificaciones que necesiten los capacitadores y coordinadores del programa barrial, sin incurrir en gastos.

FASE 3: En la evaluación destacó la plataforma e-learning Moodle la cual cumplió en un más alto puntaje ante las otras consideradas en cada uno de los parámetros especificados en la FASE 1, por lo cual fue la plataforma escogida para el presente proyecto.

FASE 4: El siguiente paso fue socializar la plataforma escogida, para poder recibir y acoplar las especificaciones de los coordinadores de cada proyecto.

- Administración del sitio

- Capacitación del manejo de recurso multimedia Moodle

- Configuración de los módulos para el ingreso de contenidos.

- Configuración para la evaluación de actividades en foros, lecciones, tareas, glosarios y talleres.

Al establecer un cuadro de responsables de cada carrera se realizó una capacitación a todos los estudiantes responsables de módulos para que puedan crear las actividades (pdfs, juegos, diapositivas, exámenes, cualquier alternativa didáctica) en las cuales se registraban calificaciones por cada módulo progresivo que se aprobaba, en base a preguntas debidamente estructuradas. Con esto se fortalece inicialmente la 
interdisciplinariedad, así como la independencia también en determinadas actividades empoderando a cada actor en su especialidad.

\section{RESULTADOS Y DISCUSIÓN}

Una vez establecida la plataforma, se organizan varios niveles de avance en las cuales tenemos el Nivel 1 de capacitación donde se tuvo un periodo de inscripciones y según se completaba cada uno de los módulos al final se obtenía un certificado como "Facilitador comunitario y en derechos humanos Nivel 1".

\section{Para otros casos se optó por una capacitación intensiva vía zoom con la siguiente}

\section{propuesta:}

- Creación de invitaciones para las capacitaciones del Nivel 1 y de formulario de inscripción mediante Google Forms.

- Habilitación de la sala en la plataforma zoom para impartir las capacitaciones en las cuales se brindó ayuda durante todas las sesiones de trabajo.

- Se socializó el material usado en las capacitaciones mediante correo electrónico y Whatsapp.

- Se implementó una evaluación en línea como requisito previo a la obtención del certificado que previó a una verificación fue enviado a sus correos electrónicos.

En el Nivel 2 se establece dictar una capacitación mediante zoom, con las siguientes actividades:

- La creación de invitaciones para este nivel y del formulario de inscripción mediante Google Forms, la creación de la sala en la plataforma zoom para impartir las capacitaciones en las cuales se dio soporte durante el tiempo de capacitaciones. De igual forma se socializó el material usado en las charlas mediante correo electrónico y Whatsapp, se creó un repositorio en línea para almacenar trabajos finales, y por último se revisó los trabajos para su aprobación y envíos de certificado de las personas aprobadas.

- Como es común en la realización de grandes proyectos que involucran a varios sectores, departamentos o en este caso facultades, la sincronización de tareas y desarrollo del cumplimiento de objetivos se torna complejo debido a la cantidad de personas que están trabajando de manera síncrona, pero cuando este trabajo es liderado por las personas indicadas con las capacidades adecuadas se logran cosas como las que ha cumplido este programa. 
- Para brindar un contexto del alcance y la magnitud del presente proyecto podemos mencionar como menos los siguientes datos que demuestran como la multidisciplinariedad es la base teórica y operativa de este.

En la Tabla 2, se presentan datos importantes del programa los cuales son importantes recalcar para visualizar el potencial que tiene y su proyección como vinculación con la sociedad.

Tabla 2 - Datos Programa Barrios de Quito

\section{BENEFICIARIOS Personas directas aproximadas: 20.000}

Ciencias Sociales y Humanas, Ciencias Aplicadas , Facultad de

Facultades Comunicación Social, Ingeniería Química, Ciencias Químicas, Jurisprudencia, Ciencias Médicas, Filosofía.

\begin{tabular}{lc}
\hline Docentes & 40 \\
\hline Estudiante & 500 \\
\hline
\end{tabular}

A esto se suman más de 20 proyectos internos del desarrollo e impartición de conocimientos, capacitaciones, planificaciones y desarrollos que estos requieren.

El Programa Barrios de Quito interactúa con más de 152 barrios ubicados en el Norte, Sur, Centro y Valles de Quito, algunos afiliados a la Federación de Barrios de Quito, otros de organizaciones territoriales como Chillogallo, la Ecuatoriana, Guamaní, el Beaterio, Chilibulo, La Magdalena, Mena 2, la Ferroviaria, Calderón, Carapungo, Cocotog, Atucucho, San Juan, Toctiuco, etc. Miles de beneficiados, cientos de estudiantes y docentes comprometidos con una realidad que debemos cambiar luego de una pandemia que ha derrumbado la economía de los moradores del D. M. de Quito y por lo cual buscamos dar alternativa desde lo académico para mejorar sus condiciones de vida, motivo por el cual se puso en funcionamiento un nuevo proyecto en la plataforma llamado "Yo Emprendo" y de igual manera con el Vicerrectorado de Investigación, Innovación y Doctorados el Proyecto “ Huella Juvenil” - en los cuales, ya se ha tenido la capacitación a los estudiantes que harán uso de la estructura tecnológica para la creación de actividades y de los diferentes módulos que definan según la temática planteada.

\section{CONCLUSIONES}

- Se evaluó varias plataformas E-learning cada una con sus ventajas y desventajas, Moodle fue la más adecuada ya que su aplicabilidad ha beneficiado la rápida implementación del programa de Barrios de Quito. 
- Se configuró la plataforma Moodle para que la implementación de los diferentes proyectos de Barrios de Quito sea más amigables y sencillas de usar.

- Se realizó con éxito la capacitación a los beneficiarios del programa Barrios de Quito que usaban por primera vez una plataforma E-learning, gracias a la participación de los estudiantes, se obtuvo una enriquecedora experiencia colaborativa reforzando las competencias profesionales cada estudiante.

- El grupo de informática brindó soporte a cada uno de los diferentes proyectos para su buen manejo y algunas actividades que requiera el grupo de programas de Barrio de Quito

\section{RECOMENDACIONES:}

- Moodle no es la única plataforma que existe, se puede hacer uso de cualquiera, sea de código abierto o privado. El fin de una evaluación de plataformas es el elegir la que más sea conveniente para el fin del programa.

- El tema que se elige en la plataforma Moodle para el diseño debe ser de igual forma seleccionado dependiendo como se quiera tener la estructura del proyecto.

- Es necesario estructurar un número de capacitaciones y horas para enseñar el uso de la plataforma, así podemos abarcar todos los temas e inquietudes que necesiten los usuarios y paralelamente un chat personalizado si fuese necesario.

- Tener un grupo que lidere y esté presente en los tiempos de capacitaciones intensivas, o virtuales de la plataforma, para ayudar en caso de necesitar soporte a los expositores.

\section{REFERENCIAS BIBLIOGRAFICAS}

Basantes, A. V. (2018). Scielo. Obtenido de https://doi. org/10.4067/S071850062018000200035

Bravo, P. C. (2006). E-learning y desarrollo de competencias:. Obtenido de E-learning y desarrollo de competencias:: https://dialnet.unirioja.es/servlet/articulo?codigo=2229192

De-La-Hoz, E. J. (2019). Metodología de Aprendizaje Automático para la Clasificación y Predicción de Usuarios en Ambientes Virtuales de Educación. Información Tecnológica. Obtenido de https://doi.org/10.4067/s0718-07642019000100247

Gallegos Elias, W. H. (2021). Herramientas virtuales para la promoción del aprendizaje emocional en estudiantes universitarios. Revista Publicando, 113-123. 
Galvis, Á. H. (2016). Desafíos del elearning y del blearning en educación superior. Universidad de los Andes, 1-5.

HERNÁNDEZ-ASCANIO, J. T.-M. (2016). El concepto de innovación social: ambitos, definiciones y alcan ces teóricos. CIRIEC-España, Revista de Economía Pública, Social y Cooperativa., 88, 165-199.

Kizilcec, R. F.-S. (2017). Self-regulated Learningstrategiespredictlearnerbehavior and goal attainment in Massive Open Online Courses. Computers \& education, 104, 18-33.

Magaña-Valladares, L. G.-R.-M.-A.-O. (2018). Capacitación de profesionales de la salud primaria en la prevención del cáncer de mama: evidencia y experiencia de México. Revista de educación sobre el cáncer , 33, 160-166.

Moneta Pizarro, A. M. (2018). Hacia un modelo estructural del e-learning. Centro Regional Universitario Córdoba IUA.

Mora-Vicarioli, F. (2019). Estado del arte de la evaluación de los aprendizajes en la modalidad del e-learning desde la perspectiva de evaluar para aprender: precisiones conceptuales. Revista Electrónica Calidad en la Educación Superior, $58-95$.

Moreno, C. P. (2016). La Economía necesita E-learning. Obtenido de La Economía necesita E-learning: http://revistas.ut.edu.co/index.php/Ideales/article/view/876/678

Pérez Pérez, S. M. (2020). Utilización de la plataforma Moodle en la disciplina Informática Médica de la carrera de Medicina. Edumecentro, 37-48.

Ramos, L. (2021). Universidad Pedagógica Nacional. Obtenido de http://repository.pedagogica.edu.co/handle/20.500.12209/13341

Requena, J. V. (2004). E-learning y desarrollo de competencias:. Obtenido de E-learning y desarrollo de competencias:: https://dialnet.unirioja.es/servlet/articulo? codigo=2229192

Salvat, B. G. (2018). La evolución del e-learning: del aula virtual a la red. RIED. Revista Iberoamericana de Educación a Distancia, 21.

Suyo-Vega, J. A.-L.-R.-B. (2021). Miradas divergentes sobre la metodología virtual universitaria. Cuadernos de desarrollo aplicados a las TIC, 69-91. 
Trovato J, T. N. (2013). Un enfoque interprofesional basado en la web para brindar educación sobre el cáncer de mama. . J Cancer Educ , 639-646.

Van Laer, S. y. (2017). In search of attributes that support self-regulation in blended learning environments. Education and Information Technologies, 1395-1454.

Verdezoto Rodríguez, R. H. (2018). Importancia de las herramientas y entornos de aprendizaje dentro de la plataforma e-learning en las universidades del Ecuador. . Edutec. Revista Electrónica De Tecnología Educativa, 68-92.

Yen, C. J.-M. (2016). A Predictor for PLE Management: Impacts of SelfRegulated Online Learning on Students' Learning Skills. . Journal of Educational Technology Development and Exchange (JETDE), 3-9. 